\title{
Approximating Minimum Steiner Point Trees in Minkowski Planes*
}

\author{
M. Brazil $\quad$ C. J. Ras $\quad$ D. A. Thomas
}

\begin{abstract}
Given a set of points, we define a minimum Steiner point tree to be a tree interconnecting these points and possibly some additional points such that the length of every edge is at most 1 and the number of additional points is minimized. We propose using Steiner minimal trees to approximate minimum Steiner point trees. It is shown that in arbitrary metric spaces this gives a performance difference of at most $2 n-4$, where $n$ is the number of terminals. We show that this difference is best possible in the Euclidean plane, but not in Minkowski planes with parallelogram unit balls. We also introduce a new canonical form for minimum Steiner point trees in the Euclidean plane; this demonstrates that minimum Steiner point trees are shortest total length trees with a certain discrete-edge-length condition.

Keywords: minimum Steiner point trees, bounded edge-length, Minkowski planes
\end{abstract}

\section{Introduction}

Given a metric space $(S, d)$ with metric $d$, a set of points $N \subseteq S$, and an $R \in \mathbb{R}$, the minimum Steiner point tree problem (MSPT problem) asks for a set $U \subset S$ and a tree connecting $N \cup U$ such that no edge is longer than $R$ and $|U|$ is minimized. Clearly we may assume that $R=1$. An optimal solution is called a $d$-MSPT, or just an MSPT if the context is clear. MSPTs have applications in the deployment and augmentation of wireless sensor networks, VLSI design, and wavelength-division multiplexing networks - see for instance [6, 11, 13, 16.

The MSPT problem was first described by Sarrafzadeh and Wong in [17], where they showed that it is NP-complete in both the $\ell_{1}$ and $\ell_{2}$ metric. Consequently a fair amount of research has been directed towards finding good heuristics. In [12] the minimum spanning tree (MST) heuristic was introduced (note that there they refer to the MSPT problem as the Steiner tree problem with minimum number of Steiner points and bounded edge length, or STP-MSPBEL). This heuristic

\footnotetext{
*This research was supported by an ARC Discovery Grant.
} 
simply subdivides all edges of an MST that are longer than one unit, resulting in an approximate MSPT solution within polynomial time. Mandoiu and Zelikovsky [14 prove that, in any metric space, the performance ratio of the MST heuristic is always one less than the maximum possible degree of a minimum-degree MST spanning points from the space. This gives an approximation ratio of four in the Euclidean plane and three in the rectilinear plane. Chen et al. [5] provide an improved approximation scheme, partly based on the MST heuristic, which has a performance ratio of three in the Euclidean plane.

The MSPT problem may be seen as a variant of the classical Steiner tree problem, which asks for a shortest tree interconnecting $N \subseteq S$ where any number of additional points may be introduced. An optimal solution to this problem is called a Steiner minimal tree ( $d$-SMT or just SMT). As $R$ tends to zero an SMT with subdivided edges becomes an optimal solution to the MSPT problem. This leads us to the question: would the SMT approximation for the MSPT problem be a practical and accurate heuristic? Certainly we do not have effective algorithms for calculating SMT's in every metric, in fact the problem is NP-hard. However, in the Euclidean plane and other fixed orientation metrics, Warme, Winter, and Zachariasen [18, 19] have developed practical, fast and optimal SMT algorithms, namely the GeoSteiner algorithms. These algorithms can comfortably solve most instances of up to a few thousand uniformly distributed terminals. However, as should be expected, it is possible to construct terminal-sets that take much longer to process; for instance, GeoSteiner cannot efficiently find an SMT when just one hundred terminals are located at the vertices of a regular square lattice in the Euclidean plane (although these instances can be solved in polynomial time by the algorithms of Brazil et al. [3]).

In this paper we define and analyze the SMT heuristic for MSPTs. We provide a small upper bound (in terms of $|N|$ ) for the performance difference of the SMT heuristic in any normed plane, and show that this bound is best possible in the Euclidean plane. We then show that, in the special case $|N|=3$, the upper bound is tight in a Minkowski plane with unit ball $B$ if and only if $B$ is not a parallelogram. For the Euclidean and rectilinear planes a brief comparison between the SMT heuristic and current best possible heuristics is given. Then we prove that the performance ratio of the SMT heuristic improves as $R$ decreases (or equivalently, as the terminals become further apart). This paper also explores the possibility of restating the Euclidean MSPT problem in terms of shortest total length, leading to a new MSPT canonical form. Finally, we state a number of strong conjectures on the relationship between the Steiner tree problem and the MSPT problem.

\section{Preliminaries}

Let $(S, d)$ be a metric space with metric $d$, and consider a set $N \subseteq S$. The Steiner tree problem asks for a shortest tree interconnecting $N$, where extra nodes $W \subset S$ are introduced if they reduce the total length. Introducing degree-one or degree-two nodes will not reduce total length, henceforth 
for the Steiner tree problem we assume all added nodes are of degree at least three. The nodes in $N$ are called terminal points and the nodes in $W$ are called Steiner points.

In general metric spaces there may be instances of the MSPT problem that have no solution; consider, for instance, the case when $N=S$ and $\min \{d(x, y): x, y \in S\}>1$. Henceforth we will assume the following: $S=\mathbb{R}^{2},|N|$ is finite, and $d$ is a norm. In other words, we will only be considering the finite MSPT problem in Minkowski planes. In our discussions we distinguish between the concept of a free node and an embedded node. In other words any tree may be considered as a topological graph structure only, or as an embedded network. Embedded nodes are denoted by bold letters (as is common when representing vectors). An embedded set of terminals admits a tree with property $P$ if there exists a tree $T$ interconnecting the terminals such that $T$ has property $P$.

Two standard techniques for shortening an embedded tree are splitting and Steiner point displacements. To split a node $v$ one disconnects two or more of the edges at $v$ and connects them instead to a new Steiner point, connected to $v$ by an extra edge. To displace a Steiner point one simply embeds it at any new point in the plane without changing the topology of the tree. If no shortening of a tree is possible when splitting or Steiner point displacements are allowed, then the tree is called a Steiner tree. Note that an SMT is always a Steiner tree. A full Steiner tree is a Steiner tree where every terminal is of degree one and every Steiner point is of degree three. A full Steiner tree has exactly $|N|-2$ Steiner points and $2|N|-3$ edges. A cherry of a full Steiner tree is the subtree induced by two terminals and their mutually adjacent Steiner point. Every full Steiner tree has at least two cherries. We refer the reader to [9] and [10] for more background on Steiner trees.

Given two points $x, y \in S$, we denote the edge $e$ between them by $e=x y$, and we use the standard notation $|e|$ to denote $d(x, y)$. Any Steiner tree can be viewed as a candidate MSPT if we simply subdivide, or bead, edges that are longer than one unit. Formally, beading is the process whereby for every edge $e$, $\lceil|e|\rceil-1$ equally spaced degree-two nodes lying on $e$ are included (along with the elements of $W$ ) in the set $U$ of extra MSPT nodes. In general, any tree can be viewed as an MSPT candidate if we partition its nodes into a set $N$ of terminals and a set $W$ of Steiner points of degree at least three, and then bead any edges that are too long. Consequently, when constructing an MSPT on a given set $N$, we are mainly concerned with finding the elements of $W$, i.e., the elements of $U$ that have degree at least three; clearly degree-one nodes will not occur in $U$ and degree-two nodes in $U$ only arise from beading. Henceforth, degree-two nodes in $U$ will not be considered as part of the topology of the MSPT. All nodes in $U$ will be referred to as beads and, specifically, the nodes in $W$ will be called Steiner beads. The procedure of constructing an SMT in order to approximate an MSPT will be referred to as the SMT heuristic.

Let $T$ be any tree with node-set partitioned into terminals $N$ and Steiner beads $W$. Let $n=|N|$. Then $T^{*}$ is the tree that results by splitting nodes of $T$ until every terminal is of degree one and every Steiner bead is of degree three (i.e., $T^{*}$ is a full Steiner tree). New nodes are not displaced from their original positions, in other words some zero edge-lengths may be introduced and the total 
length of $T$ does not change. See Figure 1 as an example; here $t$ is a degree-four terminal, $s$ is a Steiner point, and after splitting $t$ we have three zero-length edges (depicted by broken lines).
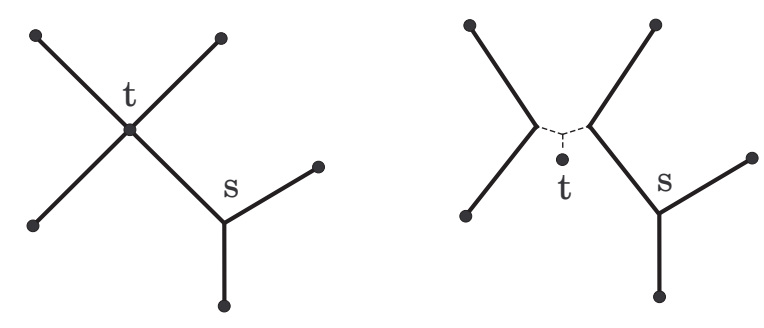

Figure 1: Conversion to a full Steiner tree.

Let the edge-set of $T^{*}$ be $E\left(T^{*}\right)=\left\{e_{1}, \ldots, e_{m}\right\}$, where $m=2 n-3$. Then the bead count of $T$ is $\operatorname{beads}(T)=|U|=n-2+\sum_{i=1}^{m}\left(\left\lceil\left|e_{i}\right|\right\rceil-1\right)=1-n+\sum_{i=1}^{m}\left\lceil\left|e_{i}\right|\right\rceil$. In other words, by considering $T^{*}$ rather than $T$ we get a formula for beads $(T)$ that does not depend on the number of Steiner beads of $T$; this formula works because every time a node is split (creating a new Steiner bead) we introduce a zero-length edge which in effect cancels the count of this Steiner bead. We can now reformulate the MSPT problem as follows. Let $N$ be a subset of $S$. Find a $W \subset S$ and a tree $T$ interconnecting $N \cup W$ such that every node in $W$ is of degree at least three and $\operatorname{beads}(T)$ is a minimum over all trees interconnecting $N$.

\section{The Upper Bound in any Normed Plane}

In this section we provide an upper bound for the performance difference of the SMT heuristic in any normed plane. Let $N$ be a set of $n$ terminals in a normed plane $\left(\mathbb{R}^{2}, d\right)$. We use $T_{\text {opt }}$ to denote an MSPT on $N$ and $T_{S}$ to denote an SMT on $N$. We need the following lemma before we prove our main result:

Lemma 1 If $i, k$ are real numbers then $\lceil i+k\rceil-\lceil i\rceil=\lceil k\rceil$ or $\lceil k\rceil-1$ (equivalently $\lfloor k\rfloor$ or $\lfloor k\rfloor+1$ ), with $\lceil i+k\rceil-\lceil i\rceil=k$ if $k$ is an integer.

Proof. Suppose that $\lceil i\rceil=i+\varepsilon_{i}$ and $\lceil k\rceil=k+\varepsilon_{k}$ where $0 \leq \varepsilon_{i}, \varepsilon_{k}<1$. Then $\lceil i+k\rceil=$ $\lceil i\rceil+\lceil k\rceil-\left\lfloor\varepsilon_{i}+\varepsilon_{k}\right\rfloor$, from which the result follows.

Suppose that $E\left(T_{\mathrm{opt}}^{*}\right)=\left\{e_{1}, \ldots, e_{m}\right\}$ and $E\left(T_{S}^{*}\right)=\left\{a_{1}, \ldots, a_{m}\right\}$. Since $T_{S}$ is a shortest total length tree interconnecting $N$, we have:

$$
\sum_{i=1}^{m}\left|a_{i}\right| \leq \sum_{i=1}^{m}\left|e_{i}\right|
$$


We can therefore partition the set $\{1, \ldots, m\}$ as follows: let $\{1, \ldots, m\}=I \cup D$ such that $\left|e_{i}\right|=\left|a_{i}\right|+p_{i}$ for $i \in I$ and $\left|e_{i}\right|=\left|a_{i}\right|-p_{i}$ for $i \in D$. Here each $p_{i}$ is a non-negative real number and the cardinality of $D$, but not $I$, may be zero. We further partition $I$ into $I_{Z}$ and $I_{Z}^{\prime}$ (where $I_{Z}$ may be empty) such that $i \in I_{Z}$ if and only if $\left|a_{i}\right|$ is an integer. We similarly partition $D$ into $D_{Z}$ and $D_{Z}^{\prime}$. From (1) it follows that $\sum_{i \in I} p_{i} \geq \sum_{i \in D} p_{i}$ - a result that is central to the proof of Proposition 3

Lemma 2 If all edges of both $T_{S}$ and $T_{\mathrm{opt}}$ have integer length, then $T_{\mathrm{opt}}$ is also an $S M T$ on $N$, and $\operatorname{beads}\left(T_{S}\right)=\operatorname{beads}\left(T_{\text {opt }}\right)$.

Proof. Let $T$ be any Steiner tree on $N$ such that all edges of $T$ have integer length, and let $L(T)$ be the total length of $T$. Then beads $(T)=L(T)-n+1$. The lemma immediately follows since $L\left(T_{\mathrm{opt}}\right) \geq L\left(T_{S}\right)$.

Proposition 3 beads $\left(T_{S}\right)$ - beads $\left(T_{\mathrm{opt}}\right) \leq \max \{2 n-4-j, 0\}$, where $j$ is the number of integerlength edges in $E\left(T_{S}^{*}\right)$.

Proof.

$$
\begin{aligned}
\operatorname{beads}\left(T_{S}\right)-\operatorname{beads}\left(T_{\mathrm{opt}}\right) & =\left[1-n+\sum_{i=1}^{m}\left\lceil\left|a_{i}\right|\right\rceil\right]-\left[1-n+\sum_{i=1}^{m}\left\lceil\left|e_{i}\right|\right\rceil\right] \\
& =\sum_{i=1}^{m}\left\lceil\left|a_{i}\right|\right\rceil-\sum_{i=1}^{m}\left\lceil\left|e_{i}\right|\right\rceil \\
& =\sum_{i \in D}\left\{\left\lceil\left|a_{i}\right|\right\rceil-\left\lceil\left|a_{i}\right|-p_{i}\right\rceil\right\}-\sum_{i \in I}\left\{\left\lceil\left|a_{i}\right|+p_{i}\right\rceil-\left\lceil\left|a_{i}\right|\right\rceil\right\} .
\end{aligned}
$$

Using Lemma 1 we obtain:

$$
\begin{aligned}
\operatorname{beads}\left(T_{S}\right)-\operatorname{beads}\left(T_{\mathrm{opt}}\right) & \leq \sum_{i \in D_{Z}}\left\lfloor p_{i}\right\rfloor+\sum_{i \in D_{Z}^{\prime}}\left(\left\lfloor p_{i}\right\rfloor+1\right)-\sum_{i \in I_{Z}}\left\lceil p_{i}\right\rceil-\sum_{i \in I_{Z}^{\prime}}\left(\left\lceil p_{i}\right\rceil-1\right) \\
& =\left|D_{Z}^{\prime}\right|+\left|I_{Z}^{\prime}\right|+\sum_{i \in D}\left\lfloor p_{i}\right\rfloor-\sum_{i \in I}\left\lceil p_{i}\right\rceil \\
& \leq m-j+\sum_{i \in D} p_{i}-\sum_{i \in I}\left\lceil p_{i}\right\rceil \\
& \leq m-j+\sum_{i \in I} p_{i}-\sum_{i \in I}\left\lceil p_{i}\right\rceil \\
& \leq m-j \\
& =2 n-3-j .
\end{aligned}
$$

We now consider a number of cases showing that either (2) or (3) is a strict inequality or beads $\left(T_{S}\right)=$ $\operatorname{beads}\left(T_{\mathrm{opt}}\right)$. Together, these imply the statement of the lemma. 
CASE 1: Suppose there exists an edge in $T_{S}$ that is longer than some edge in $T_{\text {opt }}$ and such that the difference between the lengths of the two edges is not an integer. Then there exists an assignment of labels $\left\{e_{i}\right\}$ to the edges of $T_{\text {opt }}$ and labels $\left\{a_{i}\right\}$ to the edges of $T_{S}$ such that $p_{i} \notin \mathbb{Z}$ for some $i \in D$. Hence Inequality (2) is strict.

CASE 2: If there exists an edge in $T_{S}$ that is shorter than some edge in $T_{\text {opt }}$ and such that the difference between the lengths of the two edges is not an integer, then by the same argument as in Case 1, we can assume Inequality (3) is strict.

CASE 3: The only remaining possibility is that the difference in length between each edge in $T_{S}$ and each edge in $T_{\mathrm{opt}}$ is an integer. This means there exists an $\varepsilon \in[0,1)$ such that the length of every edge in both trees is an integer plus $\varepsilon$. If $\varepsilon=0$ then $\operatorname{beads}\left(T_{S}\right)-\operatorname{beads}\left(T_{\mathrm{opt}}\right)=0$ by Lemma 2 , If $\varepsilon \neq 0$ then we can move any Steiner point in $T_{\mathrm{opt}}$ by a sufficiently small distance $(>0)$ such that the length of at least one edge changes without changing the bead count of $T_{\mathrm{opt}}$. Hence we can then apply Case 1 or 2 .

Corollary 4 beads $\left(T_{S}\right)$ - beads $\left(T_{\mathrm{opt}}\right) \leq 2 n-c-3$ where $c$ is the number of full components of $T_{S}$.

Proof. Note that every terminal $x$ of $\operatorname{degree} \operatorname{deg}(x)$ is split $\operatorname{deg}(x)-1$ times to produce $T_{S}^{*}$, i.e., each terminal $x$ produces $\operatorname{deg}(x)-1$ zero-length edges after all splits. Clearly also $c=\sum_{x \in N}\{\operatorname{deg}(x)-1\}+1$.

Corollary 5 If $T_{S}$ has at most one edge with non-integer length then beads $\left(T_{S}\right)=\operatorname{beads}\left(T_{\mathrm{opt}}\right)$.

Du et al. [5, 7] provide approximations for the MSPT problem that give performance ratios with upper bounds of three in the Euclidean plane and two in the rectilinear plane. Their algorithms are based on the MST heuristic and therefore run in polynomial time. If we rewrite our performance difference to get the bounded ratio $\frac{\operatorname{beads}\left(T_{S}\right)}{\operatorname{beads}\left(T_{\mathrm{opt}}\right)} \leq 1+\frac{2 n-4}{\operatorname{beads}\left(T_{\mathrm{opt} t}\right)}$ we see that the performance ratio of the SMT heuristic has a smaller upper bound than the heuristics of Du et al. when beads $\left(T_{\mathrm{opt}}\right)>n-2$ in the Euclidean plane, and beads $\left(T_{\text {opt }}\right)>2 n-4$ in the rectilinear plane. Since beads $\left(T_{\text {opt }}\right)$ increases as the minimum distance between any pair of terminals increases, we arrive at the intuitive fact that the performance of the SMT heuristic improves as the terminal configuration becomes more sparse. If $R$ was not fixed then we would arrive at the same result by decreasing $R$. During this limiting process the upper bound of the ratio $\frac{\operatorname{beads}\left(T_{S}\right)}{\operatorname{beads}\left(T_{M}\right)}$, where $T_{M}$ is an MST, tends towards the well-known Steiner ratio. This gives a limiting upper bound of $\frac{\operatorname{beads}\left(T_{S}\right)}{\operatorname{beads}\left(T_{M}\right)} \leq \frac{\sqrt{3}}{2}$ in the Euclidean plane, which serves as a comparison between the performances of the SMT heuristic and the standard MST heuristic.

We mention once again that the SMT heuristic does not run in polynomial time. However, for $n$ up to a few thousand nodes (uniformly distributed in a square) the GeoSteiner algorithms will produce 
solutions in reasonable running time for the Euclidean and rectilinear plane [18. This makes the SMT heuristic a tool worthy of consideration for applications where optimization is required during an initialization process (such as deployment). In fact, one should consider this heuristic for any process where the cost benefit of a more accurate algorithm justifies a possible time delay.

It should also be noted that SMTs can be approximated arbitrarily closely in polynomial time. The polynomial-time approximation scheme (PTAS) developed by Arora [1, 2] works for any norm, and allows one to construct a solution to the SMT problem that is within a factor of $1+\epsilon$ from optimality in polynomial time for any fixed $\epsilon>0$. In theory this gives a good polynomial-time heuristic for the MSPT problem, obtained by replacing the SMT by its $1+\epsilon$ approximation. There is, however, a difficulty with this approach in that the degree of the polynomial for small values of $\epsilon$ is too large to make the algorithm practical.

\section{The Euclidean Plane}

The aim of this section is to show that the performance difference from Proposition 3 is best possible in the Euclidean plane. We begin with a few definitions and preliminary results. Due to minimality of total length, any two adjacent edges of a Euclidean Steiner tree meet at an angle of at least $120^{\circ}$. This implies that the degree of any terminal is no more than 3, and the degree of any Steiner point is exactly 3. Let $T$ be a full Euclidean Steiner tree on a set of embedded terminals. To sprout new terminals from a given terminal $\mathbf{t}$ of $T$ with incident edge $e$ one replaces $\mathbf{t}$ by a Steiner point $\mathbf{s}$ and embeds two new terminals $\mathbf{t}_{1}, \mathbf{t}_{2}$ adjacent to $\mathbf{s}$ such that the two new edges $\mathbf{s t}_{1}$ and $\mathbf{s t}_{2}$ each form $120^{\circ}$ angles with $e$ and with each other - see Figure 2 We denote by $L(T)$ the total Euclidean edge length of $T$. If $N$ is a set of embedded terminals then $T_{S}$ will denote a Euclidean SMT on $N$ and $T_{\text {opt }}$ will denote a Euclidean MSPT on $N$. As usual we let $n=|N|$. The next proposition shows that we can use sprouting to create full SMTs with any given topology. It is a fundamental result and is almost certainly known, but does not appear to have been explicitly written up in the literature before now.

Proposition 6 Given any full Steiner topology, there exists a set of embedded terminals $N$ such that the SMT for $N$ has the given topology and is unique. Furthermore, such trees can be explicitly constructed for any given topology.

Proof. Let $G_{n}$ be a full Steiner topology on $n$ terminals. We will show how a suitable set of embedded terminals $N_{n}$ can be constructed by induction on $n$, where the inductive step involves sprouting new terminals. Note that the construction is trivial if $n=1,2$ or 3 . The inductive claim is as follows.

Claim: For any full Steiner topology $G_{i}$ on $i$ terminals (with $i \geq 4$ ), there exists a set of embedded terminals $N_{i}$ and a real number $f_{i}>0$ such that 

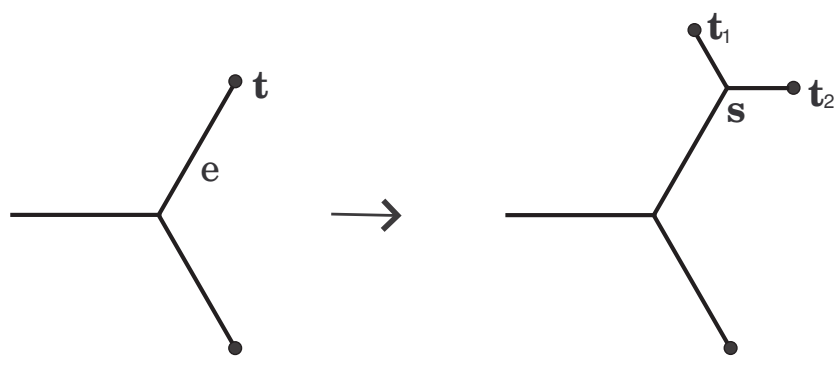

Figure 2: Sprouting new terminals.

1. an SMT, $T_{i}$, for $N_{i}$ has topology $G_{i}$, and

2. if $T_{i}^{\prime}$ is a Steiner tree for $N_{i}$ such that the topology of $T_{i}^{\prime}$ is not $G_{i}$, then $L\left(T_{i}^{\prime}\right)-L\left(T_{i}\right) \geq f_{i}$.

For the base case of the claim $(i=4)$, choose $N_{4}$ to be the four points with coordinates $( \pm 1, \pm \sqrt{3} / 2)$. It is easily checked that the SMT $T_{4}$ for $N_{4}$ has Steiner points $( \pm 1 / 2,0)$ and length 5 (see Figure 3). The shortest Steiner tree $T_{4}^{\prime}$ with a different topology is full with Steiner points $(0, \pm(\sqrt{3} / 2-1 / \sqrt{(3)}))$ and length $L\left(T_{4}^{\prime}\right)=3 \sqrt{3}$. So we can choose $f_{4}=3 \sqrt{3}-5>0$. Up to relabelling of the terminals, there is only one full topology for $i=4$, so this completes the base case.

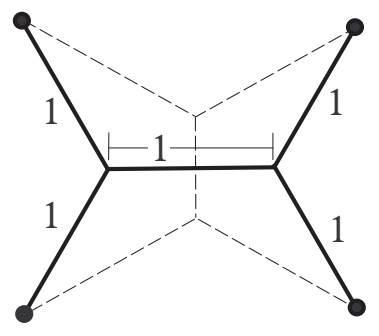

Figure 3: Base case.

We now establish the inductive step for $(i=n)$, where we assume that the claim holds for $i=n-1$. Given a full Steiner topology $G_{n}(n>4)$, this topology contains at least one cherry. Replacing such a cherry by a single terminal $t^{*}$ gives a full Steiner topology $G_{n-1}$ on $n-1$ terminals. By the inductive assumption there exists an embedded terminal set $N_{n-1}$ with unique SMT $T_{n-1}$ which has topology $G_{n-1}$ and a corresponding constant $f_{n-1}>0$. Let $\mathbf{t}$ be the embedded terminal corresponding to $t^{*}$ and create a new Steiner tree as follows.

We sprout new terminals $\mathbf{t}_{n}$ and $\mathbf{t}_{n-1}$ from $\mathbf{t}$, with $\mathbf{t}$ replaced by a Steiner point $\mathbf{s}$, such that $\left|\mathbf{s t}_{n-1}\right|=\left|\mathbf{s t}_{n}\right|=f_{n-1} / 4$. Let this new tree be $T_{n}$ with embedded terminal set $N_{n}$. By construction, $T_{n}$ has the correct topology $G_{n}$. 
Let $T_{n}^{\prime}$ be any Steiner tree (but not necessarily an SMT) on $N_{n}$ with topology not $G_{n}$. Suppose we collapse $\mathbf{t}_{n}$ and $\mathbf{t}_{n-1}$ onto the point $\mathbf{s}$ (fixing all other nodes in the network), and consider the topology $G$ of the resulting network. $G$ is a topology on $n-1$ terminals, but may be different from $G_{n-1}$, indeed $G$ is not necessarily a tree. If $G=G_{n-1}$, then $T_{n}^{\prime}$ also has the same topology as $T_{n}$, which by convexity and the fact that $T_{n}^{\prime}$ is a Steiner tree implies that $T_{n}^{\prime}=T_{n}$ (see Theorem 1.3 of [10]); this is a contradiction and hence $G \neq G_{n-1}$. It follows from this that, if we consider the network $T_{n}^{\prime} \cup\left\{\mathbf{s t}_{n}\right\}$ (which interconnects $N_{n-1}$ ), we have $L\left(T_{n}^{\prime}\right)+\left|\mathbf{s t}_{n}\right| \geq L\left(T_{n-1}\right)+f_{n-1}$. This implies that

$$
\begin{aligned}
L\left(T_{n}^{\prime}\right) & \geq L\left(T_{n-1}\right)+f_{n-1}-\left|\mathbf{s t}_{n}\right| \\
& =L\left(T_{n-1}\right)+3\left|\mathbf{s t}_{n}\right|=L\left(T_{n}\right)+\left|\mathbf{s t}_{n}\right| .
\end{aligned}
$$

Hence, we can choose $f_{n}=f_{n-1} / 4<L\left(T_{n}^{\prime}\right)-L\left(T_{n}\right)$.

The claim (and lemma) now follow. Furthermore, the iterative algorithm for constructing a suitable set of embedded terminals for any required Steiner topology is constructive with $f_{i}=(3 \sqrt{3}-5) / 4^{i-4}$ for each $i \geq 4$.

Proposition 7 Let $G_{n}$ be a full Steiner topology on $n$ terminals. There exists an embedded set of terminals $N$ in the Euclidean plane such that beads $\left(T_{S}\right)=\operatorname{beads}\left(T_{\mathrm{opt}}\right)+2 n-4$ and $T_{S}$ has topology $G_{n}$.

Proof. We construct an SMT $T_{S}$ with topology $G_{n}$ by repeatedly sprouting terminals, starting from a full Steiner tree on three terminals called the base. By the previous proposition any full Steiner topology can be produced in this way. Note that we can create a base with edges of any length by simply intersecting the end-points of three line segments at one common point such that every pair of segments forms an angle of $120^{\circ}$ (and we have complete freedom to do this since we are constructing an SMT by choosing positions for the terminals). By making the edges of the base large enough, it is clear that we can construct $T_{S}$ such that every edge-length has the form $a_{i} \pm \varepsilon_{i}$, where $a_{i}$ is an integer of order at least two and $\varepsilon_{i}$ has any predefined value between zero and one. $T_{S}$ is then converted into an MSPT by a sequence of displacements (which we describe below) of the Steiner points, where displacements do not change the original topology $G_{n}$.

In $T_{S}$, let $\mathbf{s}_{0}$ be a Steiner point adjacent to a terminal $\mathbf{t}$ and two other nodes $\mathbf{v}_{1}, \mathbf{v}_{2}$ where edgelengths are preselected as follows: $\left|\mathbf{t s}_{0}\right|=a_{1}-\varepsilon,\left|\mathbf{s}_{0} \mathbf{v}_{1}\right|=\left|\mathbf{s}_{0} \mathbf{v}_{2}\right|=b_{1}+\varepsilon_{1}$ for large integers $a_{1}, b_{1}$ and $0<\varepsilon, \varepsilon_{1}<1$. In the first step (Figure 4) we displace $\mathbf{s}_{0}$ along the line through $\mathbf{t}$ and $\mathbf{s}_{0}$ and in the direction of the vector $\overrightarrow{\mathbf{t}} \mathbf{s}_{0}$. We displace until $\left|\mathbf{t s}_{0}\right|=a_{1}-\varepsilon^{\prime}$ and $\left|\mathbf{s}_{0} \mathbf{v}_{1}\right|=\left|\mathbf{s}_{0} \mathbf{v}_{2}\right|=b_{1}-\varepsilon_{1}^{\prime}$ for some $0<\varepsilon^{\prime}, \varepsilon_{1}^{\prime}<1$. Clearly this is possible as long as we preselect $\varepsilon_{1}$ to be small enough compared to $\varepsilon$.

We now displace all other Steiner points in a depth-first or breadth-first order rooted at $\mathbf{t}$. Suppose that in the process we have reached the Steiner point $\mathbf{s}$ with parent $\mathbf{s}^{\prime}$ and children $\mathbf{u}_{1}, \mathbf{u}_{2}$. We 


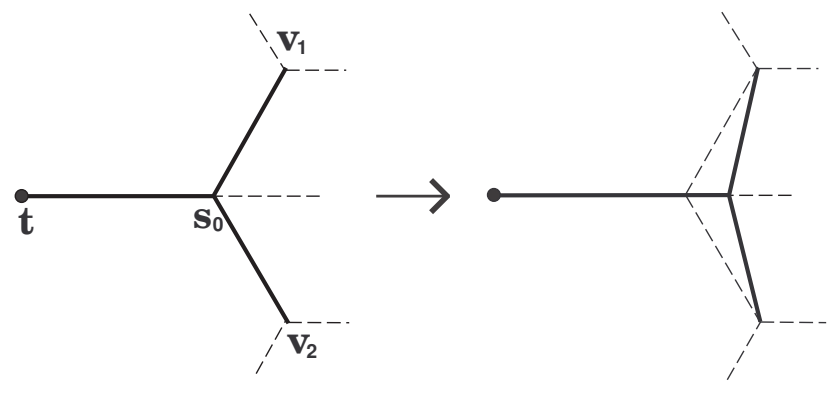

Figure 4: First step of the displacement sequence.

displace $\mathbf{s}$ along the line through $\mathbf{s}$ and the point $\mathbf{p}$ and in the direction $\overrightarrow{\mathbf{p s}}$, where $\mathbf{p}$ is the position $\mathbf{s}^{\prime}$ had before its displacement; see Figure 5. If $\left|\mathbf{s s}^{\prime}\right|=a-\varepsilon_{1}$ then we preselect $\left|\mathbf{s u}_{1}\right|=\left|\mathbf{s u}_{2}\right|=b+\varepsilon_{2}$ for $0<\varepsilon_{2}<1$. We select $\varepsilon_{2}$ small enough so that the displacement of $\mathbf{s}$ produces the lengths $\left|\mathbf{s s}^{\prime}\right|=a-\varepsilon_{1}^{\prime}$ and $\left|\mathbf{s u}_{1}\right|=\left|\mathbf{s u}_{2}\right|=b-\varepsilon_{2}^{\prime}$, for some $0<\varepsilon_{1}^{\prime}, \varepsilon_{2}^{\prime}<1$. We continue this process until we have displaced all Steiner points. Call the resultant tree $T$. Note that the edges of $T_{S}$ were preselected so that one edge has length $a_{1}-\varepsilon$ and every other edge $e_{i}$ has length $b_{i}+\varepsilon_{i}$. After all displacements the first edge has length $a_{1}-\varepsilon^{\prime}$ and every other $e_{i}$ has length $b_{i}-\varepsilon_{i}^{\prime}$. Clearly then $\operatorname{beads}\left(T_{S}\right)=\operatorname{beads}(T)+2 n-4$ and $T$ is an MSPT.

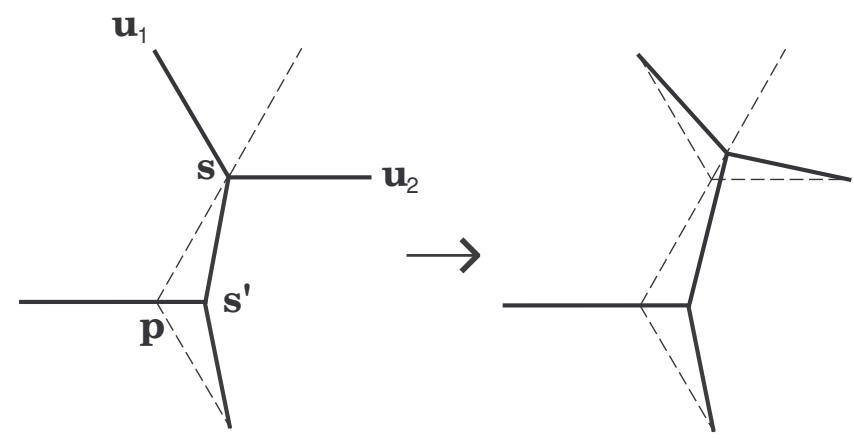

Figure 5: General step of the displacement sequence.

\section{$5 \quad$ Minkowski Planes}

A Minkowski plane is a two-dimensional real normed space $M=\left(\mathbb{R}^{2},\|\cdot\|_{M}\right)$ with unit ball $B=\{\mathbf{x}$ : $\left.\|\mathbf{x}\|_{M} \leq 1\right\}$. We denote the metric induced by $M$ by $d_{M}(\mathbf{x}, \mathbf{y})=\|\mathbf{x}-\mathbf{y}\|_{M}$. Examples of Minkowski planes include the Euclidean plane and the rectilinear plane, where the unit balls are the circle and the $45^{\circ}$ rotated square respectively. The unit ball of a Minkowski plane is always convex, centrally symmetric and bounded in the Euclidean norm. Conversely, any such convex body is the unit ball 
of a Minkowski plane. The boundary of a ball $B$ is denoted by bd $(B)$ and its interior by $\operatorname{int}(B)$.

The question arises as to whether the upper bound from Proposition 3 is best possible in all Minkowski planes. In the three-terminal case we show that the upper bound can be improved for a given Minkowski plane if and only if the unit ball is a parallelogram. Well-known Minkowski planes with this property are the $L_{1}$ (rectilinear) and $L_{\infty}$ planes.

Let $N=\left\{\mathbf{t}_{i}: i=1,2,3\right\}$ be a set of embedded terminals and let $T_{S}$ be a $d_{M}$-SMT on $N$. Many of the propositions below will refer to $T_{S}^{*}$ instead of $T_{S}$ in order to maintain generality. Recall that this convention may lead to zero-length edges, and consequently also to balls of zero radius.

Throughout this section we let $L$ denote a Minkowski plane with a parallelogram unit ball. The corresponding metric is denoted by $d_{L}$. If $\mathbf{t}$ is a terminal point in the plane, we denote by $l_{1}(\mathbf{t})$ and $l_{2}(\mathbf{t})$ the Euclidean straight lines passing through $\mathbf{t}$ and parallel to the major diagonal and minor diagonal, respectively, of the parallelogram defining the unit ball of $L$. Minkowski balls $\left\{B_{i}: i=1,2,3\right\}$ tessellate at $\mathbf{x}$ if $B_{i} \cap B_{j}$ is a point or a Euclidean line segment whenever $i \neq j$, and $\bigcap B_{i}=\{\mathbf{x}\}$. The point $\mathbf{x}$ is called a tessellation point of $N$ if there exists a set of balls $\left\{B_{i}: i=1,2,3\right\}$, with $B_{i}$ centered at $\mathbf{t}_{i}$, that tessellate at $\mathbf{x}$. The next proposition is a generalization of a well-known result ( $c f$. [8]) on three-terminal rectilinear SMTs.

Proposition 8 Let $N=\left\{\mathbf{t}_{i}: i=1,2,3\right\}$ be a set of embedded terminals. Then there exists a $d_{L}-S M T T_{S}$ on $N$ such that the Steiner point of $T_{S}^{*}$ coincides with the intersection of the median of $\left\{l_{1}\left(\mathbf{t}_{i}\right)\right\}$ and the median of $\left\{l_{2}\left(\mathbf{t}_{i}\right)\right\}$.

Proof. Let $\mathbf{x}$ be a point in the plane. We wish to minimize the function $f=\left|\mathbf{t}_{1} \mathbf{x}\right|+\left|\mathbf{t}_{2} \mathbf{x}\right|+\left|\mathbf{t}_{3} \mathbf{x}\right|$ where all inequalities $\left|\mathbf{t}_{i} \mathbf{x}\right|+\left|\mathbf{t}_{j} \mathbf{x}\right| \geq\left|\mathbf{t}_{i} \mathbf{t}_{j}\right|$, with $1 \leq i<j \leq 3$, hold by the triangle inequality. Therefore a minimum would occur if $\left|\mathbf{t}_{i} \mathbf{x}\right|+\left|\mathbf{t}_{j} \mathbf{x}\right|=\left|\mathbf{t}_{i} \mathbf{t}_{j}\right|$ for every $1 \leq i<j \leq 3$; equivalently, a minimum would occur if the balls $\left\{B_{i}: i=1,2,3\right\}$, with $B_{i}$ centered at $\mathbf{t}_{i}$ and of radius $\left|\mathbf{t}_{i} \mathbf{x}\right|$, tessellate at $\mathbf{x}$. We show that this happens if $\mathbf{x}$ is the intersection of the median of $\left\{l_{1}\left(\mathbf{t}_{i}\right)\right\}$ and the median of $\left\{l_{2}\left(\mathbf{t}_{i}\right)\right\}$.

Suppose, without loss of generality, that $l_{1}\left(\mathbf{t}_{2}\right)$ is the median of $\left\{l_{1}\left(\mathbf{t}_{i}\right)\right\}$ and $l_{2}\left(\mathbf{t}_{3}\right)$ is the median of $\left\{l_{2}\left(\mathbf{t}_{i}\right)\right\}$, and let $\mathbf{x}$ be the intersection of these two lines. Let $l_{0}$ be the line through $\mathbf{x}$ and parallel to the side of $B_{3}$ that intersects $B_{2}$ at $\mathbf{x}$ only - see Figure 6. Then $\mathbf{t}_{1}$ must lie on the opposite side of $l_{0}$ to $\mathbf{t}_{2}$ and $\mathbf{t}_{3}$ (since $l_{1}\left(\mathbf{t}_{2}\right)$ and $l_{2}\left(\mathbf{t}_{3}\right)$ are medians). Therefore $B_{1} \cap B_{2} \subset l_{0}$ and $B_{1} \cap B_{3} \subset l_{0}$ and the result follows.

Corollary 9 Let $N=\left\{\mathbf{t}_{i}: i=1,2,3\right\}$ be a set of embedded terminals and let $T_{S}$ be a $d_{L}-S M T$ on $N$. Then the Steiner point of $T_{S}^{*}$ is the unique tessellation point of $N$.

Proof. The set of linear equations $\left|\mathbf{t}_{i} \mathbf{x}\right|+\left|\mathbf{t}_{j} \mathbf{x}\right|=\left|\mathbf{t}_{i} \mathbf{t}_{j}\right|$, for $1 \leq i<j \leq 3$, has a unique solution. 


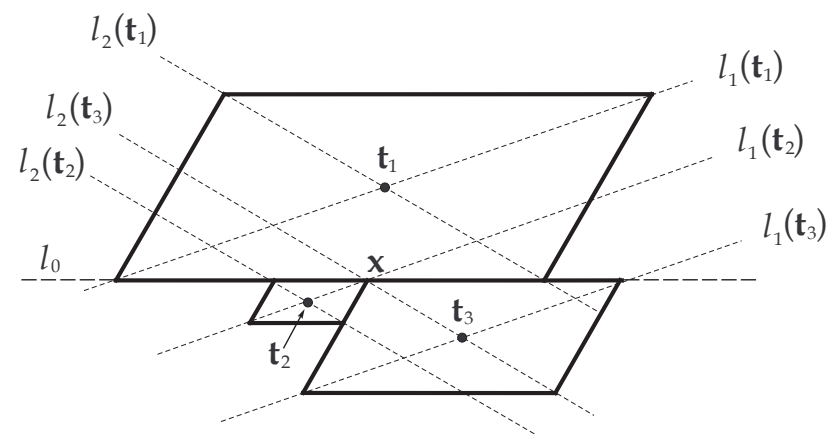

Figure 6: Median diagonals $l_{1}\left(\mathbf{t}_{2}\right)$ and $l_{2}\left(\mathbf{t}_{3}\right)$ intersect at Steiner point $\mathbf{x}$.

Given three terminals in the plane and a unit ball parallelogram $B$, the enclosing diagonalized parallelogram is the smallest parallelogram whose sides are parallel to the major and minor diagonals of $B$ and which includes all the terminals on its boundary. The next result also has an analogue in the rectilinear plane ( $c f .[8]$ ).

Corollary 10 The total length of a three-terminal $d_{L}-S M T$ is equal to half the perimeter of the enclosing diagonalized parallelogram.

The following proposition is the first main result of this section. It shows that the upper bound from Proposition 3 with $n=3$ is not strict for parallelogram-based Minkowski planes.

Proposition 11 Let $T_{S}$ be a $d_{L}-S M T$ and let $T_{\mathrm{opt}}$ be a $d_{L}-M S P T$ on the embedded terminals $\left\{\mathbf{t}_{i}\right.$ : $i=1,2,3\}$. Then $\operatorname{beads}\left(T_{S}\right) \leq \operatorname{beads}\left(T_{\mathrm{opt}}\right)+1$.

Proof. Suppose that the Steiner point of $T_{S}^{*}$ is $\mathbf{s}$ and its edges are $a_{i}=\mathbf{t}_{i} \mathbf{s}$ for $i \in\{1,2,3\}$. Let $\mathbf{s}^{\prime}$ be the Steiner bead of $T_{\mathrm{opt}}^{*}$ and let $e_{i}=\mathbf{t}_{i} \mathbf{s}^{\prime}$ for $i \in\{1,2,3\}$. Since $\mathbf{s}$ is the tessellation point of $\left\{\mathbf{t}_{i}\right\}$, at most one inequality from $\left|e_{i}\right|<\left|a_{i}\right|, i \in\{1,2,3\}$ can be true (note that if none of these inequalities are true then $\operatorname{beads}\left(T_{S}\right)=\operatorname{beads}\left(T_{\mathrm{opt}}\right)$, and we are done). Suppose w.l.o.g that $\left|e_{1}\right|<\left|a_{1}\right|$. Let $p_{1}=\left|a_{1}\right|-\left|e_{1}\right|$ and let $p_{i}=\left|e_{i}\right|-\left|a_{i}\right|$ for $i=\{2,3\}$.

Claim: $p_{1} \leq \min \left\{p_{2}, p_{3}\right\}$.

Clearly $\left|a_{1}\right|+\left|a_{3}\right|=\left|\mathbf{t}_{1} \mathbf{t}_{3}\right|$ since $\mathbf{t}_{1}, \mathbf{s}, \mathbf{t}_{3}$ is a shortest path between $\mathbf{t}_{1}$ and $\mathbf{t}_{3}$. Also, by using the triangle inequality in $\triangle \mathbf{t}_{1} \mathbf{s}^{\prime} \mathbf{t}_{3}$ we obtain $\left|\mathbf{t}_{1} \mathbf{t}_{3}\right| \leq\left|e_{1}\right|+\left|e_{3}\right|$. Therefore $\left|a_{1}\right|+\left|a_{3}\right| \leq\left(\left|a_{1}\right|-p_{1}\right)+$ $\left(\left|a_{3}\right|+p_{3}\right)$ so that $p_{1} \leq p_{3}$. Similarly, $p_{1} \leq p_{2}$ and this proves the claim.

We now have:

$$
\operatorname{beads}\left(T_{S}\right)-\operatorname{beads}\left(T_{\mathrm{opt}}\right)=\sum_{i=1}^{3}\left\lceil\left|a_{i}\right|\right\rceil-\sum_{i=1}^{3}\left\lceil\left|e_{i}\right|\right\rceil
$$




$$
\begin{aligned}
& =\left\lceil\left|a_{1}\right|\right\rceil-\left\lceil\left|e_{1}\right|\right\rceil-\sum_{i \in\{2,3\}}\left\{\left\lceil\left|e_{i}\right|\right\rceil-\left\lceil\left|a_{i}\right|\right\rceil\right\} \\
& =\left\lceil\left|e_{1}\right|+p_{1}\right\rceil-\left\lceil\left|e_{1}\right|\right\rceil-\sum_{i \in\{2,3\}}\left\{\left\lceil\left|e_{i}\right|\right\rceil-\left\lceil\left|e_{i}\right|-p_{i}\right\rceil\right\} \\
& \leq\left\lceil p_{1}\right\rceil-\sum_{i \in\{2,3\}}\left\{\left\lceil p_{i}\right\rceil-1\right\} \\
& \leq 0-\left\lceil\max \left\{p_{2}, p_{3}\right\}\right\rceil+2 \quad\left(\text { since } 0<p_{1} \leq \min \left\{p_{2}, p_{3}\right\}\right) \\
& <2 .
\end{aligned}
$$

To prove a converse of the previous proposition we first show that Corollary 9 is unique to Minkowski planes with parallelogram unit balls. We find that some three-terminal sets in Minkowski planes with hexagon unit balls do have tessellation points, but that this is not true in general. For any non-parallelogram based Minkowski plane we then construct a three-terminal example that achieves the upper bound from Proposition 3

Two points on the boundary of a ball $B$ form a diametric pair if the Euclidean straight line passing through the points also passes through the center of $B$. A point $\mathbf{z}$ on a ball $B_{i}$ is equivalent to a point $\mathbf{z}^{\prime}$ on a ball $B_{j}$ if and only if, by translating $B_{i}$ so that its center coincides with the center of $B_{j}$, it is possible to rescale $B_{i}$ so that $\mathbf{z}$ coincides with $\mathbf{z}^{\prime}$. Let $\left\{\mathbf{t}_{i}: i=1,2,3\right\}$ be a set of embedded terminals with tessellation point $\mathbf{s}$ in an arbitrary non-parallelogram-based Minkowski plane $M$, and suppose that the balls $\left\{B_{i}\right\}$ tessellate at $\mathbf{s}$.

Lemma 12 Suppose that $B_{i} \cap B_{j}=\{\mathbf{s}\}$ for some $i, j \in\{1,2,3\}$. Then the point that forms a diametric pair with $\mathbf{s}$ on $B_{i}$ is equivalent to $\mathbf{s}$ when considered as a point on $B_{j}$.

Proof. This follows from the convexity and central symmetry of the balls.

As a consequence of the previous lemma there exist distinct $i, j, k \in\{1,2,3\}$ such that $B_{i} \cap B_{j}$ and $B_{i} \cap B_{k}$ are Euclidean line segments (as opposed to single points only corresponding to s). Suppose w.l.o.g that $B_{1} \cap B_{2}$ and $B_{1} \cap B_{3}$ are line segments.

Lemma $13 B_{2} \cap B_{3}$ is also a line segment.

Proof. With the aim of producing a contradiction we assume that $B_{2} \cap B_{3}=\{\mathbf{s}\}$. Note that there exist exactly four maximal-length line segments in $\bigcup\left\{\mathrm{bd}\left(B_{i}\right)\right\}$ that have $\mathbf{s}$ as an endpoint. We list these segments in any clockwise order as $\left\{S_{i}: 0 \leq i \leq 3\right\}$. Since $\mathbf{s}$ forms a diametric pair on $B_{2}$ and $B_{3}, S_{i}$ and $S_{(i+2) \bmod 4}$ have the same gradient for any $i \in\{0, \ldots, 3\}$. By central symmetry, the only possible balls that can produce such a configuration of line segments are parallelograms, which is a contradiction. 
A point $\mathbf{x}$ on $\operatorname{bd}(B)$ is called a corner if the intersection of any neighborhood of $\mathbf{x}$ with $\operatorname{bd}(B)$ is not a Euclidean straight line segment.

Lemma 14 The point $\mathbf{s}$ is a corner of every member of $\left\{B_{i}\right\}$.

Proof. Clearly $\mathbf{s}$ is a corner of at least two members of $\left\{B_{i}\right\}$. Suppose, for a contradiction, that $\mathbf{s}$ is not a corner of some $B_{i}$. In this case there exist exactly three maximal-length line segments in $\bigcup\left\{\operatorname{bd}\left(B_{i}\right)\right\}$ that have $\mathbf{s}$ as an endpoint. Furthermore, exactly two of these segments have the same gradient. By central symmetry such a configuration of line segments can only be produced by parallelogram balls.

By combining the previous lemmas, we know there exist exactly three maximal-length line segments in $\bigcup\left\{\mathrm{bd}\left(B_{i}\right)\right\}$ that have $\mathbf{s}$ as an endpoint, all with distinct gradients. From this fact and central symmetry we conclude the following lemma:

Lemma 15 The balls $\left\{B_{i}\right\}$ are hexagons.

We also need the following two lemmas which follow directly from results by Martini, Swanepoel and Weiss [15.

Lemma 16 Suppose that $\mathbf{s}$ is a degree-three Steiner point of a $d_{M}$-SMT on embedded terminal set $\left\{\mathbf{t}_{i}: i=1,2,3\right\}$, where $\mathbf{s}$ does not coincide with a terminal. Then $\mathbf{s}$ is also a Steiner point of the terminal set $\left\{\mathbf{t}_{i}^{\prime}\right\}$ where $\mathbf{t}_{i}^{\prime}$ is any point lying on the Euclidean ray with origin s and passing through $\mathbf{t}_{i}$.

Lemma 17 There exists a set $\left\{\mathbf{t}_{i}: i=1,2,3\right\}$ of embedded terminals such that some $d_{M}$-SMT on $\left\{\mathbf{t}_{i}\right\}$ has a degree-three Steiner point that does not coincide with a terminal.

We can now prove the final proposition of this section. If the unit ball defining $M$ is not a hexagon then, by using the previous two lemmas, we can construct a critical $d_{M}$-SMT. A critical $d_{M}$-SMT on an embedded terminal set $\left\{\mathbf{t}_{i}: i=1,2,3\right\}$ has the following properties:

1. The Steiner point does not coincide with a terminal: i.e., the Steiner point is of degree three and there are no edges of zero length,

2. Each edge $e_{i}$ has length $a_{i}+\varepsilon_{i}$ where $a_{i}$ is an integer and $0<\varepsilon_{i}<1$ has any predefined value,

3. The balls centered at the terminals and meeting the Steiner point do not tessellate.

Otherwise, if $M$ 's unit ball is a hexagon we first use the previous two lemmas to find a terminal set satisfying properties (1) and (2). We then destroy the tessellation property (if necessary) by 
performing a rotational displacement around the Steiner point of one of the terminals. Let $T_{S}$ be a critical $d_{M}$-SMT on $\left\{\mathbf{t}_{i}\right\}$. The final result of this section is a converse to Proposition 11,

Proposition $18 \operatorname{beads}\left(T_{S}\right)=\operatorname{beads}\left(T_{\mathrm{opt}}\right)+2$

Proof. By Properties (1) and (3) we may displace the Steiner point s into a region corresponding to the intersection of the interiors of two balls, say int $\left(B_{1}\right) \cap \operatorname{int}\left(B_{2}\right)$. By Property $(2)$ we can preselect each $\varepsilon_{i}$ so that after displacement we have $\left|\mathbf{t}_{1} \mathbf{s}\right|<a_{1},\left|\mathbf{t}_{2} \mathbf{s}\right|<a_{2}$ and $\left|\mathbf{t}_{3} \mathbf{s}\right| \leq a_{3}+1$.

We conclude this section with two conjectures. Let $N$ be a set of $n$ terminals in a Minkowski plane $M$ with unit ball $B$, and let $T_{S}$ and $T_{\text {opt }}$ be a $d_{M}$-SMT and $d_{M}$-MSPT on $N$ respectively. Suppose first that $B$ is a parallelogram. Let $\mathbf{s}^{\prime}$ be the Steiner point of some cherry of $T_{S}$, and let $\mathbf{u}_{1}, \mathbf{u}_{2}$ be terminals adjacent to $\mathbf{s}^{\prime}$. The proof of Proposition 11 implies that a displacement of $\mathbf{s}^{\prime}$ can shorten at most one of $\mathbf{s}^{\prime} \mathbf{u}_{1}, \mathbf{s}^{\prime} \mathbf{u}_{2}$. Since every Steiner tree has a minimum of two cherries, displacements of Steiner points can shorten at most $2 n-5$ edges of $T_{S}$ (note, of course, that it may be possible to shorten up to $2 n-4$ edges of $T_{S}$ if we change its topology).

Conjecture 19 The upper bound beads $\left(T_{S}\right)$ - beads $\left(T_{\mathrm{opt}}\right) \leq 2 n-4$ is tight if and only if $B$ is not a parallelogram.

Conjecture 20 The upper bound beads $\left(T_{S}\right)$ - beads $\left(T_{\mathrm{opt}}\right) \leq 2 n-5$ is tight if and only if $B$ is a parallelogram.

\section{A Canonical Form for Euclidean MSPTs}

Throughout this section we only consider Euclidean MSPTs. In general there are many possible ways to embed an MSPT in Euclidean space. Here we introduce a canonical form for MSPTs (over all possible embeddings) which allows us to reformulate the MSPT problem as that of finding a shortest total length tree in which almost all edges have integer length. Understanding this canonical form provides a valuable first step towards finding an efficient exact algorithm for the problem (like the canonical forms for Steiner trees in fixed-orientation metrics [4, and those used in the previously mentioned GeoSteiner algorithms for rectilinear Steiner trees). In other words, we show that in order to find MSPTs, it suffices to explore a class of trees with strong structural restrictions. The canonical form we describe is also interesting from a more theoretical point of view as it gives an insight into the geometry of MSPTs.

An MSPT where every terminal is of degree one and every Steiner bead is of degree three is called a full MSPT. This term refers to MSPTs that have this property "naturally", i.e., not through a splitting process. Recall that a full MSPT contains $n-2$ Steiner beads and $2 n-3$ edges. The 
Steiner bead of a cherry will be referred to as a cherry bead. A level-region of a function $f$ is a set of points satisfying $f=k$ for some constant $k$.

Lemma 21 Let $N$ be a set of three embedded terminals admitting a full MSPT. Then there exists an MSPT on $N$ such that at least two of its edges are of integer length.

Proof. Let $T_{\mathrm{opt}}$ be a full MSPT on the embedded terminal set $N=\left\{\mathbf{t}_{i}: i=1,2,3\right\}$ and let $\mathbf{s}$ be the Steiner bead. Then beads $\left(T_{\mathrm{opt}}\right)$ is the minimum value of the function $f=\left\lceil\left|\mathbf{t}_{1} \mathbf{s}\right|\right\rceil+\left\lceil\left|\mathbf{t}_{2} \mathbf{s}\right|\right\rceil+\left\lceil\left|\mathbf{t}_{3} \mathbf{s}\right|\right\rceil-2$, where the position of $\mathbf{s}$ is variable. The level-region $k=\left\lceil\left|\mathbf{t}_{1} \mathbf{s}\right|\right\rceil+\left\lceil\left|\mathbf{t}_{2} \mathbf{s}\right|\right\rceil+\left\lceil\left|\mathbf{t}_{3} \mathbf{s}\right|\right\rceil-2$, where $k$ is a positive integer, consists of regions that are bounded by at least one and at most six integerradius circular arcs. Since $\mathbf{s}$ does not correspond to a terminal, the region $L(\mathbf{s})$ containing $\mathbf{s}$ must be bounded by at least two arcs. Displacing $\mathbf{s}$ to coincide with an intersection point of the arcs bounding $L(\mathbf{s})$ will lead to an MSPT of the desired form; see Figure 7

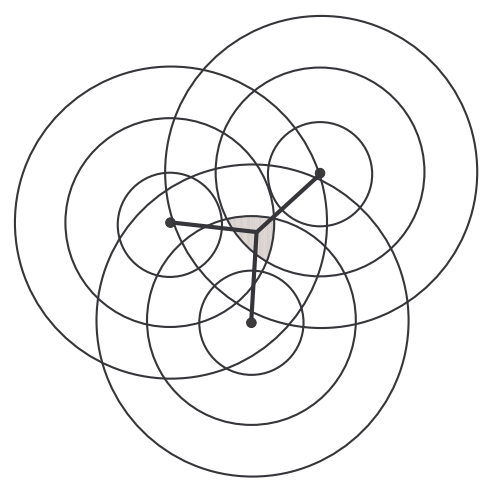

Figure 7: Level-regions of $f$.

Definition 22 A tree $T$ connecting $n$ embedded terminals and some Steiner points is called $\mathbb{Z}$-packed if $T$ has a full Steiner topology and at least $2 n-4$ of its edges are of integer length.

Proposition 23 Let $N$ be a set of three embedded terminals in the Euclidean plane admitting a full $M S P T$. Then a shortest total length $\mathbb{Z}$-packed tree on $N$ is an MSPT.

Proof. By the previous lemma, $N$ must admit a $\mathbb{Z}$-packed MSPT. Note that Proposition 3 still holds if we modify it slightly by constraining $T_{\text {opt }}$ to be $\mathbb{Z}$-packed and by letting $T_{S}$ be a shortest $\mathbb{Z}$-packed tree. Corollary 5 now gives us our result.

We wish to generalize the previous result to any number of terminal points, but for this we need another condition. If two edges of an MSPT are incident to the same Steiner bead and are collinear then these edges are said to form a Steiner bond. An MSPT $T$ on a set $N$ of embedded terminals is 
called bond-free if every MSPT on $N$ with the same topology as $T$ is free of Steiner bonds. Figure 8 provides an example of an MSPT that is not bond-free; the fact that the depicted tree is an MSPT on the three solid nodes follows from Proposition 3 once it is noted that the SMT on the same terminals has 4 beads.

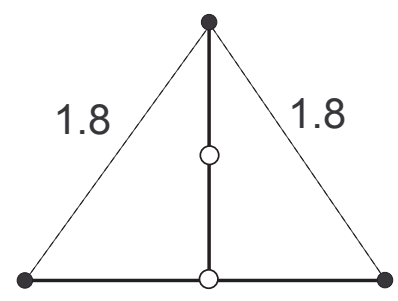

1.8

Figure 8: An MSPT that is not bond-free.

Lemma 24 Let $N$ be a set of four embedded terminals admitting a bond-free full MSPT $T_{\mathrm{opt}}$. Then it is possible to find an MSPT on $N$, with the same topology as $T_{\mathrm{opt}}$, such that all edges incident to terminals are of integer length.

Proof. Let $N=\left\{\mathbf{t}_{i}: i=1, . ., 4\right\}$ be the terminal set for $T_{\mathrm{opt}}$ and let $\mathbf{s}_{1}, \mathbf{s}_{2}$ be the Steiner beads of $T_{\text {opt }}$ with $\mathbf{s}_{1}$ adjacent to $\mathbf{t}_{1}$ and $\mathbf{t}_{2}$. Let $T_{0}$ be the subtree of $T_{\text {opt }}$ induced by the nodes $\mathbf{t}_{1}, \mathbf{t}_{2}, \mathbf{s}_{1}, \mathbf{s}_{2}$. Clearly $T_{0}$ is an MSPT on the nodes $\mathbf{t}_{1}, \mathbf{t}_{2}, \mathbf{s}_{2}$. By fixing the position of $\mathbf{s}_{2}$ we convert $T_{0}$ into a $\mathbb{Z}$-packed tree by displacing $\mathbf{s}_{1}$. We now fix $\mathbf{s}_{1}$ at its new position and displace $\mathbf{s}_{2}$ until the subtree induced by $\mathbf{t}_{3}, \mathbf{t}_{4}, \mathbf{s}_{1}, \mathbf{s}_{2}$ is $\mathbb{Z}$-packed. The modified $T_{\mathrm{opt}}$ must now have at least three integer length edges. Suppose that the edges $\mathbf{t}_{1} \mathbf{s}_{1}, \mathbf{s}_{1} \mathbf{s}_{2}, \mathbf{t}_{3} \mathbf{s}_{2}$ have integer lengths (the other cases are handled similarly). We fix $\mathbf{s}_{2}$ and displace $\mathbf{s}_{1}$ along the circle centered at $\mathbf{t}_{1}$ and of radius $\left|\mathbf{t}_{1} \mathbf{s}_{1}\right|$. Note that the smallest value of $\left|\mathbf{s}_{1} \mathbf{s}_{2}\right|$ occurs when $\mathbf{s}_{1}$ is displaced until it reaches the line connecting $\mathbf{t}_{1}$ and $\mathbf{s}_{2}$. Therefore displacement until $\left|\mathbf{t}_{2} \mathbf{s}_{1}\right|$ is an integer is possible due to the bond-free condition; see Figure 9. We fix the position of $\mathbf{s}_{1}$ and repeat the process for $\mathbf{s}_{2}$.

A caterpillar is a tree with the property that the removal of its degree-one nodes results in a path.

Lemma 25 Let $N$ be a set of $n \geq 4$ embedded terminals admitting a bond-free full MSPT $T_{\mathrm{opt}}$ and suppose that $T_{\mathrm{opt}}$ is a caterpillar. Let $\mathbf{s}_{0}$ be a cherry bead of $T_{\mathrm{opt}}$ connected to another Steiner bead $\mathbf{s}_{1}$. If $T_{\mathrm{opt}}$ is bond-free then there exists a $\mathbb{Z}$-packed MSPT on $N$ with the same topology as $T_{\mathrm{opt}}$ such that every edge, other than possibly $\mathbf{s}_{0} \mathbf{s}_{1}$, has integer length.

Proof. This follows readily from repeated application of the previous lemma.

Let $T$ be a non-caterpillar full MSPT rooted at two terminals connected to a cherry bead $\mathbf{r}$, and let $\mathbf{s}$ be any Steiner bead of $T$ with children $\mathbf{v}_{1}, \mathbf{v}_{2}$. Then $\mathbf{s}$ is called a junction of $T$ if, for each 


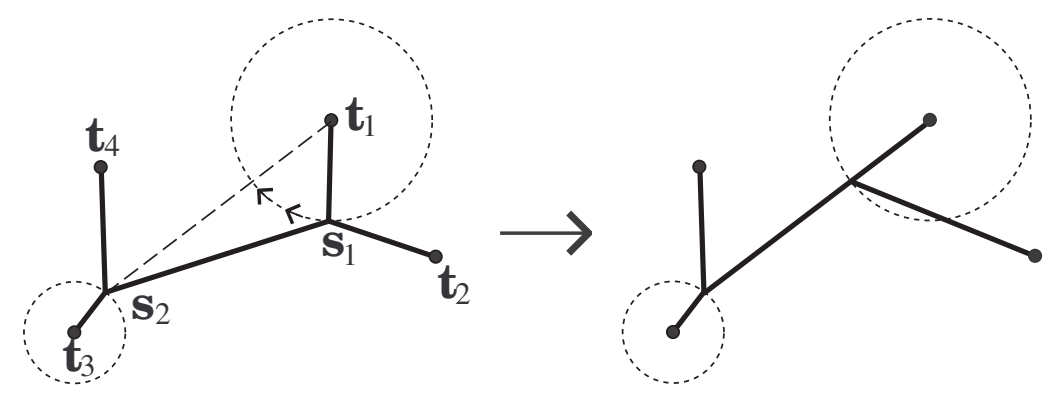

Figure 9: Bond creation.

$i \in\{1,2\}$, the subtree induced by $\mathbf{s}, \mathbf{v}_{i}$ and all descendants of $\mathbf{v}_{i}$ (if they exist) is a caterpillar. A Steiner bead is a maximal junction if it is a junction but its parent is not a junction - see Figure 10

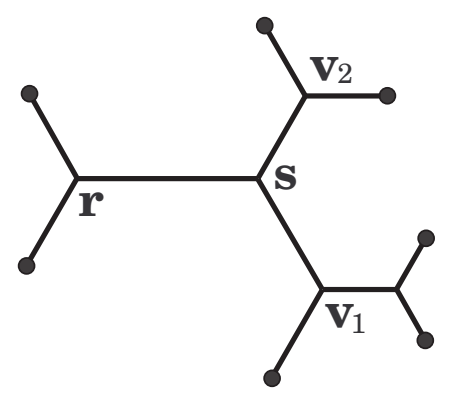

Figure 10: Maximal junction s.

Lemma 26 Let $N$ be a set of $n \geq 4$ embedded terminals admitting a bond-free full MSPT. Then it is possible to find an MSPT, say T, on $N$ such that at most one of its edges are of non-integer length. Furthermore, either all T's edges will be of integer length, or we will be able to choose which internal edge of $T$ has non-integer length.

Proof. Root $T_{\text {opt }}$ at two terminals connected to a cherry bead $\mathbf{r}$. Let $\mathbf{s}$ be a maximal junction of $T_{\mathrm{opt}}$ with children $\mathbf{v}_{1}, \mathbf{v}_{2}$ and for each $i \in\{1,2\}$ let $T_{i}$ be the subtree of $T_{\mathrm{opt}}$ induced by $\mathbf{s}, \mathbf{v}_{1}, \mathbf{v}_{2}$, the parent of $\mathbf{s}$ and the descendants of $\mathbf{v}_{i}$. Note that $T_{i}$ is a caterpillar. By fixing the positions of the parent of $\mathbf{s}$ and the child of $\mathbf{s}$ not equal to $\mathbf{v}_{i}$, and applying the previous lemma, we force all edges of $T_{i}$ (except possibly $\mathbf{s v}_{i}$ ) to be of integer length. We do this for all maximal junctions of $T_{\mathrm{opt}}$. For the resultant tree, say $T$, we now consider $\mathbf{v}_{1}$ and $\mathbf{v}_{2}$ as terminals and ignore the subtrees induced by the descendants of $\mathbf{v}_{1}$ and $\mathbf{v}_{2}$ (similarly for other junctions). We select the maximum junctions with respect to $T$ and continue the process. Once there are no junctions left (i.e., only a caterpillar remains) we force all edges, except possibly the edge between $\mathbf{r}$ and its child, to be of integer length. 
Repeated application of Lemma 24 now allows us to choose which internal edge should possibly be of non-integer length, i.e., we now "move" the non-integer property to any other internal edge.

Proposition 27 Let $N$ be any set of terminals in the Euclidean plane admitting a bond-free full $M S P T T_{\mathrm{opt}}$. Then a shortest total length $\mathbb{Z}$-packed tree on $N$ is an MSPT.

Proof. This follows, as before, from the previous result and from Corollary 5 after a slight modification to Proposition 3 ,

\section{Concluding Remarks and Conjectures}

We suspect that, at least in the Euclidean case, the performance difference $2 n-4$ of the SMT heuristic can be improved by supplementing it with an algorithm that involves relatively small displacements of the Steiner points. The question is: by how much can we improve the performance? If for any set of embedded terminals it is possible to find an MSPT with the same topology (or a degeneracy thereof) as an SMT on the terminals, then it would be theoretically possible to improve the performance to optimality in this way. If an SMT $T_{S}$ is not full then certainly the topology of an MSPT on the same terminals is generally not a degeneracy of $T_{S}$ (i.e., an MSPT topology cannot be obtained simply by collapsing edges of $T_{S}$ ). Consider for instance the embedded terminals $\left\{\mathbf{t}_{i}: i=1,2,3\right\}$ in the Euclidean plane where $\angle \mathbf{t}_{1} \mathbf{t}_{2} \mathbf{t}_{3}=120^{\circ}$ and $\left|\mathbf{t}_{1} \mathbf{t}_{2}\right|=\left|\mathbf{t}_{2} \mathbf{t}_{3}\right|=5.1$ units. Clearly the SMT $T_{S}$ on $\left\{\mathbf{t}_{i}\right\}$ is not full and $\operatorname{beads}\left(T_{S}\right)=10$. However, $\operatorname{beads}\left(T_{\mathrm{opt}}\right)=9$ as shown in Figure 11

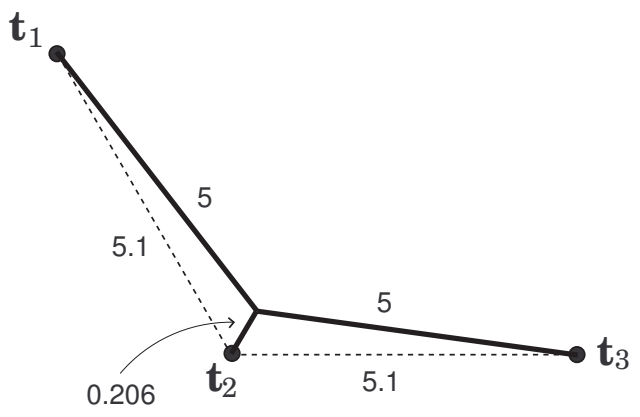

Figure 11: $T_{\text {opt }}$ with nine beads.

To conclude we now state some conjectures that we hope will inspire further research into the relationship between SMTs and MSPTs. Let $N$ be a set of terminals embedded in the Euclidean plane and let $T_{S}$ be an SMT on $N$.

Conjecture 28 If $T_{S}$ is full then there exists an MSPT on $N$ that is a degeneracy of $T_{S}$. 
This conjecture implies that an algorithm based on displacing Steiner points of an SMT has the potential for generating optimal MSPTs for a very large class of terminal configurations. The next conjecture would allow such an algorithm to run in polynomial time.

Conjecture 29 Let $G$ be any tree topology on $N$ where Steiner points are of degree at least three. Then finding a tree $T$ on $N$ with the same topology as $G$ and minimizing beads $(T)$ can be done in polynomial time.

Acknowledgement. The authors wish to thank Jamie Evans for partaking in many fruitful discussions during the development of this paper. We would also like to thank the referees for their insightful comments.

\section{References}

[1] S. Arora, Polynomial time approximation schemes for Euclidean traveling salesman and other geometric problems, Journal of the ACM 45 (1998), 753-782.

[2] S. Arora, Approximation schemes for NP-hard geometric optimization problems: A survey, Math Program Ser B 97 (2003), 43-69.

[3] M. Brazil, J.H. Rubinstein, D.A. Thomas, J.F. Weng, and N.C. Wormald, Minimal Steiner trees for rectangular arrays of lattice points, J Combin Theory Series A 79 (1997), 181-208.

[4] M. Brazil, D. A. Thomas, J. F. Weng, and M. Zachariasen, Canonical forms and algorithms for Steiner trees in uniform orientation metrics, Algorithmica 44 (2006), 281-300.

[5] D. Chen, D.-Z. Du, X.-D. Hu, G.-H. Lin, L. Wang, and G. Xue, Approximations for Steiner trees with minimum number of Steiner points, Theoretical Computer Science 262 (2001), 83-99.

[6] X. Cheng, D.-Z. Du, L. Wang, and B. Xu, Relay sensor placement in wireless sensor networks, Wireless Networks 14 (2008), 347-355.

[7] D.-Z. Du and X. Hu, Steiner tree problems in computer communication networks, World Scientific Publishing Company, Singapore, 2008.

[8] R.L. Francis, A note on the optimum location of new machines in existing plant layouts, J Indust Eng 14 (1963), 57-59.

[9] E.N. Gilbert and H.O. Pollak, Steiner minimal trees, SIAM J Appl Math 16 (1968), 1-29.

[10] F.K. Hwang, D.S. Richards, and P. Winter, The Steiner tree problem, Annals of Discrete Mathematics 53, Elsevier Science Publishers B V, Amsterdam, 1992. 
[11] C.-S. Li, F.F. Tong, C.J. Georgiou, and M. Chen, Gain equalization in metropolitan and wide area optical networks using optical amplifers, 13th Proc IEEE INFOCOM, Toronto, Canada, June 1994, pp. 130-137.

[12] G.-H. Lin and G.L. Xue, Steiner tree problem with minimum number of Steiner points and bounded edge-length, Inform Process Lett 69 (1999), 53-57.

[13] B. Lu, J. Gu, X. Hu, and E. Shragowitz, Wire segmenting for buffer insertion based on RSTPMSP, Theoretical Computer Science 262 (2001), 257-267.

[14] I.I. Mandoiu and A.Z. Zelikovsky, A note on the MST heuristic for bounded edge-length Steiner trees with minimum number of Steiner points, Inform Process Lett 75 (2000), 165-167.

[15] H. Martini, K.J. Swanepoel, and G. Weiss, The Fermat-Torricelli problem in normed planes and spaces, Journal of Optimization Theory and Applications 115 (2002), 283-314.

[16] B. Ramamurthy, J. Iness, and B. Mukherjee, Minimizing the number of optical amplifiers needed to support a multi-wavelength optical LAN/MAN, Proc IEEE INFOCOM, 16th Ann Joint Conf IEEE Computer and Communications Societies, Kobe, Japan, April 1997, pp. 261-268.

[17] M. Sarrafzadeh and C.K. Wong, Bottleneck Steiner trees in the plane, IEEE Trans Comput 41 (1992), 370-374.

[18] D.M. Warme, P. Winter, and M. Zachariasen, "Exact algorithms for plane Steiner tree problems: A computational study", Advances in Steiner Trees, D.-Z. Du, J. M. Smith, and J. H. Rubinstein (Editors), Kluwer Academic Publishers, Boston, 2000, pp. 81-116.

[19] P. Winter and M. Zachariasen, Euclidean Steiner minimum trees: An improved exact algorithm, Networks 30 (1997), 149-166. 